\title{
Modification of the magnetic properties of Co films grown on MgO (100) by treatment with $\mathrm{NaOH}$ solution
}

\author{
Hao-liang Liu, ${ }^{1}$ Tom Hauffman, ${ }^{2}$ Katrien Herdewyn, ${ }^{1}$ Herman Terryn, ${ }^{2}$ Margriet Van Bael, ${ }^{1}$ and Chris Van Haesendonck ${ }^{1, *}$ \\ ${ }^{1}$ Solid State Physics and Magnetism Section, KU Leuven, Celestijnenlaan 200 D, BE-3001 Leuven, Belgium \\ ${ }^{2}$ Department of Materials and Chemistry, Research Group Electrochemical and Surface Engineering (SURF), Vrije Universiteit Brussel, \\ Pleinlaan 2, BE-1050 Brussels, Belgium
}

(Received 23 July 2014; revised manuscript received 1 October 2014; published 20 October 2014)

\begin{abstract}
We investigated the surface morphology and magnetic properties of Co films grown on $\mathrm{MgO}$ (100) substrates before and after treatment with a $\mathrm{NaOH}$ solution. The surface morphology evolves from a continuous film into isolated hexagonal nanodisks resulting from the formation of cobalt hydroxide. In the as-grown Co films the magnetic anisotropy behaves as a superposition of fourfold magnetocrystalline anisotropy and uniaxial magnetic anisotropy (UMA), and the magnetization reversal proceeds by two-step domain-wall motion. After the $\mathrm{NaOH}$ treatment the UMA is reduced and the magnetization reversal appears to be dominated by magnetization rotation. In addition, the exchange bias effect, which in the as-deposited films results from the presence of a cobalt oxide layer, disappears after the $\mathrm{NaOH}$ treatment, and can be reinduced by annealing the sample. The observed significant changes in the magnetic properties can be related to the disappearance and reappearance of a cobalt oxide top layer.
\end{abstract}

DOI: 10.1103/PhysRevB.90.134414

\section{INTRODUCTION}

Magnetic properties of metallic and semiconducting thinfilm magnets, including, in particular, magnetic anisotropy and the exchange bias (EB) effect, are crucial for the design of spintronic devices [1,2]. In order to obtain the desired characteristics for applications, various methods have been employed to manipulate the magnetic anisotropy and magnetization reversal mechanism [3-6]. The surface morphology of a magnetic thin film can, e.g., be modified by steps in the substrate, oblique incidence growth, and ion sputtering, which generate an additional uniaxial magnetic anisotropy (UMA) originating from dipolar interactions caused by anisotropic surface roughness or Néel type interface symmetry breaking $[3,7,8]$. The additional UMA is able to manipulate to a certain extent the magnetic anisotropy and magnetization reversal of magnetic thin films. On the other hand, the EB effect is used to pin the magnetization and establish a reference magnetization direction for tunneling magnetoresistance based reading heads and nonvolatile memory. The EB effect results from the exchange coupling at an antiferromagnet/ferromagnet interface [9-11]. $\mathrm{CoO}$ is the most popular antiferromagnetic material, and the $\mathrm{CoO} / \mathrm{Co}$ interface can be generated in a convenient way by oxidation of a Co film in an oxygen atmosphere. The surface morphology of Co films can also be modified by relying on chemical reactions. Electrochemical oxidation of $\mathrm{Co}$ in $\mathrm{NaOH}$ solution results in the formation of $\mathrm{Co}(\mathrm{OH})_{2}$ and $\mathrm{CoOOH}[12,13]$, and the latter compounds can be transformed into $\mathrm{CoO}$ or $\mathrm{Co}_{3} \mathrm{O}_{4}$ by annealing at the appropriate temperature [14].

Previously, we found that the magnetic anisotropy in Fe grown on $\mathrm{MgO}$ (100) reveals a superposition of fourfold symmetry of magnetocrystalline anisotropy and UMA $[8,15]$. The magnetic anisotropy can be manipulated to a certain extent by inducing a UMA with controllable strength and orientation, and the magnetization reversal mechanism changes from

*chris.vanhaesendonck@fys.kuleuven.be
PACS number(s): 75.60.Jk, 75.30.Gw

$180^{\circ}$ domain-wall nucleation to two successive domain-wall nucleations. Moreover, a pronounced EB and a related training effect (a decrease of pinning upon consecutive field cycling) have been studied in polycrystalline $\mathrm{Co}$ films with a $\mathrm{CoO}$ top layer formed by in situ oxidation [16]. It has been predicted that high-quality epitaxial $\mathrm{Co} / \mathrm{MgO}$ or $\mathrm{Co}$ alloy/MgO layers can considerably improve the tunneling magnetoresistance characteristics [17]. In the present work, we epitaxially grow Co films on $\mathrm{MgO}$ (100) oriented substrates. The magnetic properties of the Co films are significantly modified by a treatment with $\mathrm{NaOH}$ solution. Before, only minor modifications of the magnetic anisotropy and of the magnetization reversal have been reported $[3,4,15]$. We attribute the significant modifications to the formation of different cobalt compounds during the chemical reactions resulting from the treatment with $\mathrm{NaOH}$.

\section{EXPERIMENT}

The Co films were deposited on $\mathrm{MgO}$ (001) oriented substrates by molecular beam epitaxy at a base pressure of $2 \times 10^{-10}$ mbar. After annealing the substrate at $600{ }^{\circ} \mathrm{C}$ for $1 \mathrm{~h}$ to remove the adsorbed gases on the surface, nominal $5 \mathrm{~nm}$ thick Co films were deposited at a rate of $0.49 \mathrm{~nm} / \mathrm{min}$ while keeping the substrate at $300^{\circ} \mathrm{C}$. Subsequently, a $2 \mathrm{~nm}$ thick $\mathrm{CoO}$ layer was formed on top of the Co by applying a partial oxygen pressure of $10^{-3}$ mbar for 2 min [18]. In order to modify the surface morphology, the Co films were immersed in $\mathrm{NaOH}$ solution for $1 \mathrm{~min}$ at $70^{\circ} \mathrm{C}$. Next, the samples were rinsed with MilliQ water (Millipore Corp., Billerica, MA, USA) and dried with compressed dry nitrogen. Finally, the treated Co films were annealed in an ultrahigh vacuum chamber at $300^{\circ} \mathrm{C}$ for $1 \mathrm{~h}$. The surface morphology of the Co films before and after treatment was investigated by atomic force microscopy (AFM). Additionally, the crystalline structure of the films was identified by $\mathrm{x}$-ray diffraction (XRD). The magnetic anisotropy and magnetization reversal were characterized by magneto-optical Kerr magnetometry (MOKE) and magnetic force microscopy (MFM) at room 


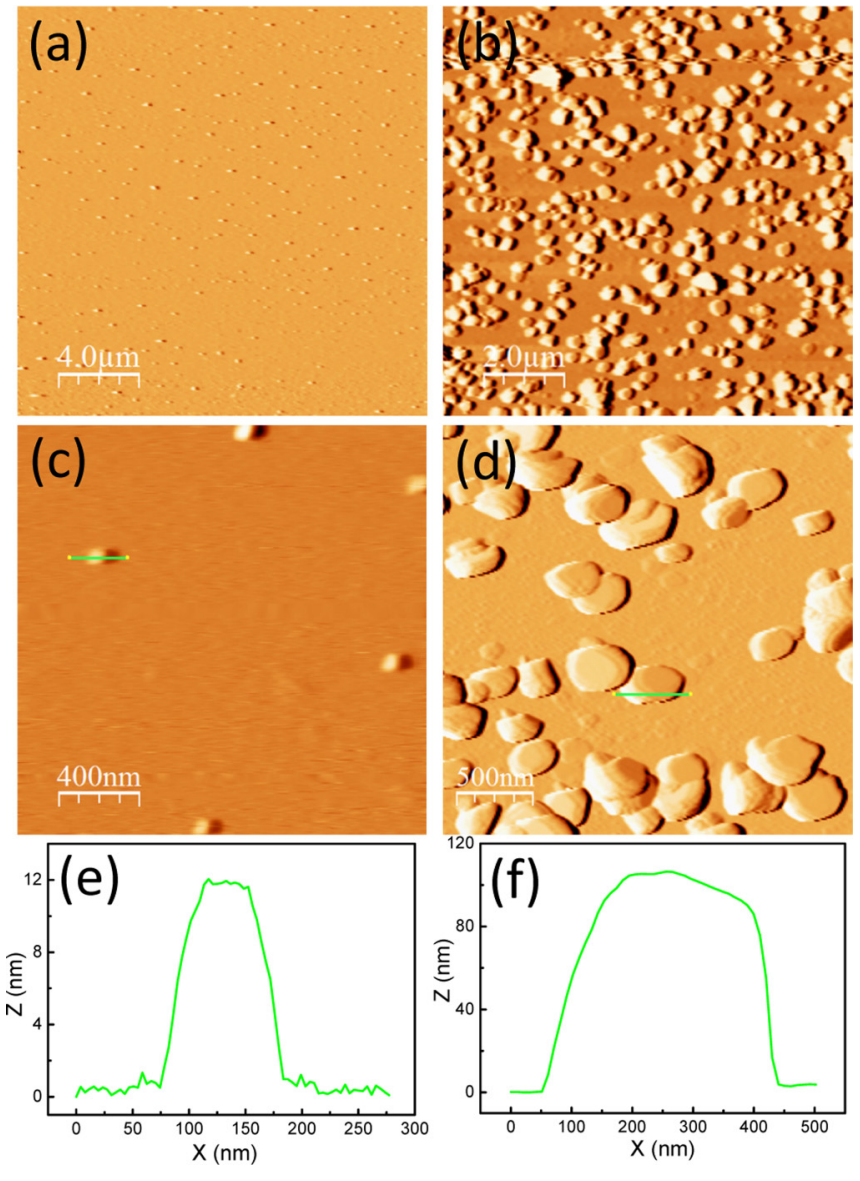

FIG. 1. (Color online) AFM topography images at different magnifications of (a) and (c) an as-grown Co film on $\mathrm{MgO}$ (100) and (b) and (d) this Co film after treatment with $\mathrm{NaOH}$ solution. (e) presents the line profile of the Co island marked by the green line in (c). (f) presents the line profile of the hexagonal $\mathrm{Co}(\mathrm{OH})_{2}$ nanodisk indicated by the green line in $(\mathrm{d})$.

temperature. The EB effect was measured from room temperature down to $10 \mathrm{~K}$ with a superconducting quantum interference device (SQUID) based magnetometer with an applied cooling field of $400 \mathrm{mT}$ along the [100] direction of the substrate. Finally, x-ray photoelectron spectroscopy (XPS) measurements were performed with a PHI 5600ci setup (Perkin Elmer, Waltham, MA, USA), using a voltage of $10 \mathrm{kV}$ and a power of $200 \mathrm{~W}$. All narrow scan spectra were acquired with a pass energy of $23.5 \mathrm{eV}$.

\section{RESULTS AND DISCUSSIONS}

Figure 1(a) presents a large scale AFM image of an as-grown Co film on $\mathrm{MgO}$ (100). The film is quite flat with a root-mean-square (rms) roughness around $0.8 \mathrm{~nm}$ for a scanned area of $400 \mu \mathrm{m}^{2}$, except for the presence of a number of very high islands that stick out of the surface and are probably resulting from strain relief [19]. In Fig. 1(c) we present an enlarged AFM view of the Co film. The islands are randomly distributed across the surface with a nearest neighbor distance of at least $700 \mathrm{~nm}$. The typical width of the islands is around $100 \mathrm{~nm}$, while their typical height is around $10 \mathrm{~nm}$,

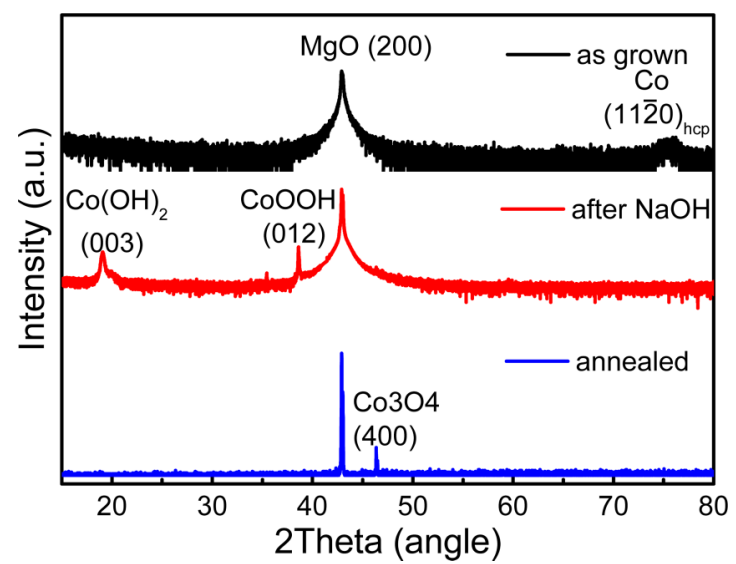

FIG. 2. (Color online) XRD diffraction patterns of the as-grown Co film on $\mathrm{MgO}$ (100), this Co film after treatment with $\mathrm{NaOH}$ solution, and this same Co film after annealing.

as indicated by the line profile of an island in Fig. 1(e). In Fig. 1(b) we present the surface morphology of the Co film after treatment with $\mathrm{NaOH}$ solution. The film now contains a number of much larger size islands, resulting in an average rms roughness around $80 \mathrm{~nm}$ for a scanned area of $100 \mu \mathrm{m}^{2}$. The higher magnification AFM image of the treated film in Fig. 1(d) reveals the presence of nearly hexagonally shaped islands which we refer to as nanodisks or nanosheets [20]. Some of these nanodisks or nanosheets are overlapping with each other. The line profile of one nanodisk is presented in Fig. 1(f). The width of the nanodisk is about $400 \mathrm{~nm}$ and the height is around $100 \mathrm{~nm}$. The two types of islands, which appear in the as-grown and in the treated Co films, respectively, are totally different. This points towards a possibly different chemical composition and structure. An additional annealing step does not further affect the surface morphology.

In order to further identify the structure of the Co films, XRD measurements were done before and after $\mathrm{NaOH}$ treatment, as illustrated in Fig. 2. The diffraction peak of the Co $(11 \overline{2} 0)_{\text {hcp }}$ planes is consistent with the one reported in Ref. [21]. After treatment with the $\mathrm{NaOH}$ solution, the Co $(11 \overline{2} 0)_{\text {hcp }}$ diffraction peak disappears, while other peaks appear that can be related to the presence of $\mathrm{Co}(\mathrm{OH})_{2}$ and $\mathrm{CoOOH}$. This can be accounted for by the occurrence of the following chemical reactions $[12,22]$ :

$$
\begin{aligned}
\mathrm{Co}+2 \mathrm{OH}^{-} & \rightarrow \mathrm{Co}(\mathrm{OH})_{2}+2 e^{-}, \\
\mathrm{Co}(\mathrm{OH})_{2}+\mathrm{OH}^{-} & \rightarrow \mathrm{CoOOH}+\mathrm{H}_{2} \mathrm{O}+e^{-} .
\end{aligned}
$$

Hexagonally shaped $\mathrm{Co}(\mathrm{OH})_{2}$ and $\mathrm{CoOOH}$ nanostructures have been synthesized before by various methods [22,23]. We therefore link the appearance of the hexagonal nanodisks in Fig. 1(d) to the formation of $\mathrm{Co}(\mathrm{OH})_{2}$ and $\mathrm{CoOOH}$. By annealing the chemically treated Co films at $300^{\circ} \mathrm{C}$ for $1 \mathrm{~h}$, a $\mathrm{Co}_{3} \mathrm{O}_{4}$ related diffraction peak appears, while the $\mathrm{Co}(\mathrm{OH})_{2}$ and $\mathrm{CoOOH}$ related peaks disappear. A possible mechanism is provided by the following temperature induced chemical reaction $[14,22]$ :

$$
\begin{aligned}
12 \mathrm{CoOOH} & \rightarrow 4 \mathrm{Co}_{3} \mathrm{O}_{4}+\mathrm{O}_{2}+6 \mathrm{H}_{2} \mathrm{O}, \\
6 \mathrm{Co}(\mathrm{OH})_{2}+\mathrm{O}_{2} & \rightarrow 2 \mathrm{Co}_{3} \mathrm{O}_{4}+6 \mathrm{H}_{2} \mathrm{O} .
\end{aligned}
$$



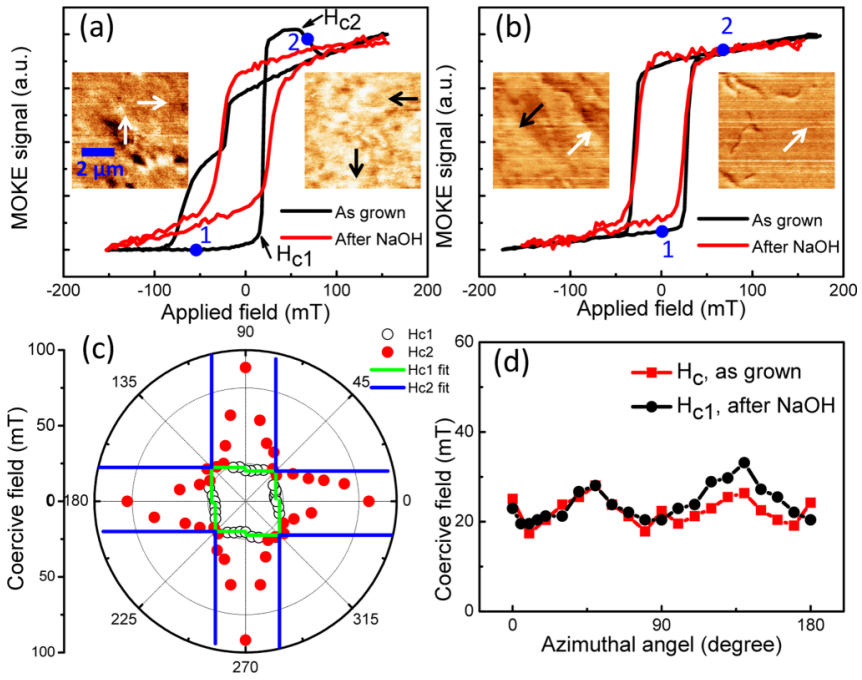

FIG. 3. (Color online) Magnetic hysteresis loops measured by MOKE along (a) the [100] direction and (b) the [110] direction of the $\mathrm{MgO}$ (100) substrate for the as-grown Co film and for this Co film after treatment with $\mathrm{NaOH}$ solution. The insets of (a) and (b) present the magnetic domain structure measured by MFM at the applied magnetic fields labeled in the respective hysteresis loops for the as-grown Co film. (c) The azimuthal angular dependence of the switching field in the hysteresis loops for the as-grown Co film. The straight lines correspond to the fitted curves based on the model for two-jump domain-wall motion. (d) The azimuthal angular dependence of the coercive field in the hysteresis loops for the Co film after treatment with $\mathrm{NaOH}$ solution.

Our experiments indicate that the surface morphology as well as the crystalline structure of the Co films are significantly modified by the treatment with $\mathrm{NaOH}$ solution. As a result, the magnetic properties are also modified to a large extent. Figure 3(a) presents the magnetic hysteresis loops of an as-grown and a treated Co film along the $\mathrm{MgO}$ [100] substrate direction as measured by MOKE. A distinct two-jump domain-wall motion reversal process appears in the hysteresis loop of the as-grown Co film. The asymmetry of the hysteresis loop is a consequence of the second-order Kerr effect [15,24]. The signature of the two-jump domain-wall motion as well as the asymmetry disappear for the hysteresis loop after the treatment with the $\mathrm{NaOH}$ solution. In the insets of Fig. 3(a) we present the MFM images of the magnetic domains of the as-grown Co film before (left inset) and after (right inset) the two-jump domain-wall nucleation. The magnetization direction (indicated by the arrows) is along the [110] and [1110] directions before the two-jump domain-wall motion and along the [110] and [110] directions after the two-jump domain-wall motion. This indicates that each jump of the domain-wall motion is close to $90^{\circ}$. The hysteresis loops along the $\mathrm{MgO}$ [110] substrate direction are similar for the as-grown and the treated Co film, as illustrated in Fig. 3(b). In the insets of Fig. 3(b) we present the magnetic domain images of the as-grown Co film with the applied magnetic field indicated on the hysteresis loop. The magnetization direction is always parallel to the [110] direction. The switching fields occurring for the hysteresis loops of the as-grown Co film are collected in Fig. 3(c). The fourfold symmetry of the magnetic anisotropy is clearly observed with the hard axis along the $\langle 100\rangle$ directions and the easy axis along the $\langle 110\rangle$ directions. The previously introduced model for $90^{\circ}$ domain-wall motion can be employed to fit the azimuthal angular dependence of the switching fields [25]. The total free energy density is given by

$$
\begin{aligned}
E= & K_{1} \cos \left(\theta+\frac{\pi}{4}\right)^{2} \cos \left(\theta-\frac{\pi}{4}\right)^{2}+K_{u} \sin \left(\theta+\frac{\pi}{4}\right)^{2} \\
& -\mu_{0} M_{S} H \cos (\theta-\varphi),
\end{aligned}
$$

where $\theta$ and $\varphi$ denote the angle of magnetization and the angle of the applied field with respect to the $\mathrm{MgO}$ [100] direction, respectively. The first term and the second term represent the magnetocrystalline anisotropy and the uniaxial magnetic anisotropy, respectively. We adopt the approximation that for low fields the local minima of the total energy will

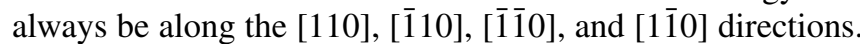
The azimuthal angular dependence of the switching field for the two-jump domain-wall motion results in the following expression for the lower switching field,

$$
\begin{aligned}
& H_{c 1}=\frac{\varepsilon_{90^{\circ}} \mp K_{u}}{ \pm \sqrt{2} M_{S} \cos \varphi}, \quad 0 \leq \varphi \leq \frac{\pi}{4} \quad \text { or } \quad \frac{3 \pi}{4} \leq \varphi \leq \pi, \\
& H_{c 1}=\frac{\varepsilon_{90^{\circ}} \mp K_{u}}{\sqrt{2} M_{S} \sin \varphi}, \quad \frac{\pi}{4} \leq \varphi \leq \frac{\pi}{2} \quad \text { or } \quad \frac{\pi}{2} \leq \varphi \leq \frac{3 \pi}{4},
\end{aligned}
$$

and the higher switching field,

$$
\begin{aligned}
& H_{c 2}=\frac{\varepsilon_{90^{\circ}} \pm K_{u}}{\sqrt{2} M_{S} \sin \varphi}, \quad 0 \leq \varphi \leq \frac{\pi}{4} \quad \text { or } \quad \frac{3 \pi}{4} \leq \varphi \leq \pi \\
& H_{c 2}=\frac{\varepsilon_{90^{\circ}} \pm K_{u}}{ \pm \sqrt{2} M_{S} \cos \varphi}, \quad \frac{\pi}{4} \leq \varphi \leq \frac{\pi}{2} \quad \text { or } \quad \frac{\pi}{2} \leq \varphi \leq \frac{3 \pi}{4} .
\end{aligned}
$$

$\varepsilon_{90^{\circ}}$ denotes the $90^{\circ}$ domain-wall pinning energy. The azimuthal angular dependence of the lower switching field can be nicely fitted, but the fitting fails for the higher switching fields. For the relatively high switching fields, especially close to the hard axis, the $90^{\circ}$ domain-wall motion model is no longer suitable since the magnetization rotation has a nonnegligible contribution. The deviation between the fitted and experimental values for the higher switching fields is related to the particular dependence of the switching field on the domainwall angle [24]. By relying on the fitting of the lower switching fields we obtain the following magnetic parameters: $K_{u}=$ $2.38 \times 10^{3} \mathrm{~J} / \mathrm{m}^{3}$ and $\varepsilon_{90^{\circ}}=4.20 \times 10^{4} \mathrm{~J} / \mathrm{m}^{3}$. The magnetocrystalline anisotropy constant $K_{1}=6.50 \times 10^{4} \mathrm{~J} / \mathrm{m}^{3}$ is determined by the location where the hysteresis loop measured by the MOKE signal changes sense [15]. We note that the experimental data cannot be fitted by one-jump domain-wall motion even near the easy axis, which is different from the situation in other systems [15,25]. The azimuthal angular dependence of the coercive fields for the treated Co film is presented in Fig. 3(d). The results are quite comparable to the results for the lower switching fields before the treatment. The fourfold symmetry of the magnetocrystalline anisotropy of Co still exists, while the UMA strength decreases (see the difference in coercivity between $45^{\circ}$ and $135^{\circ}$ ). The origin of 

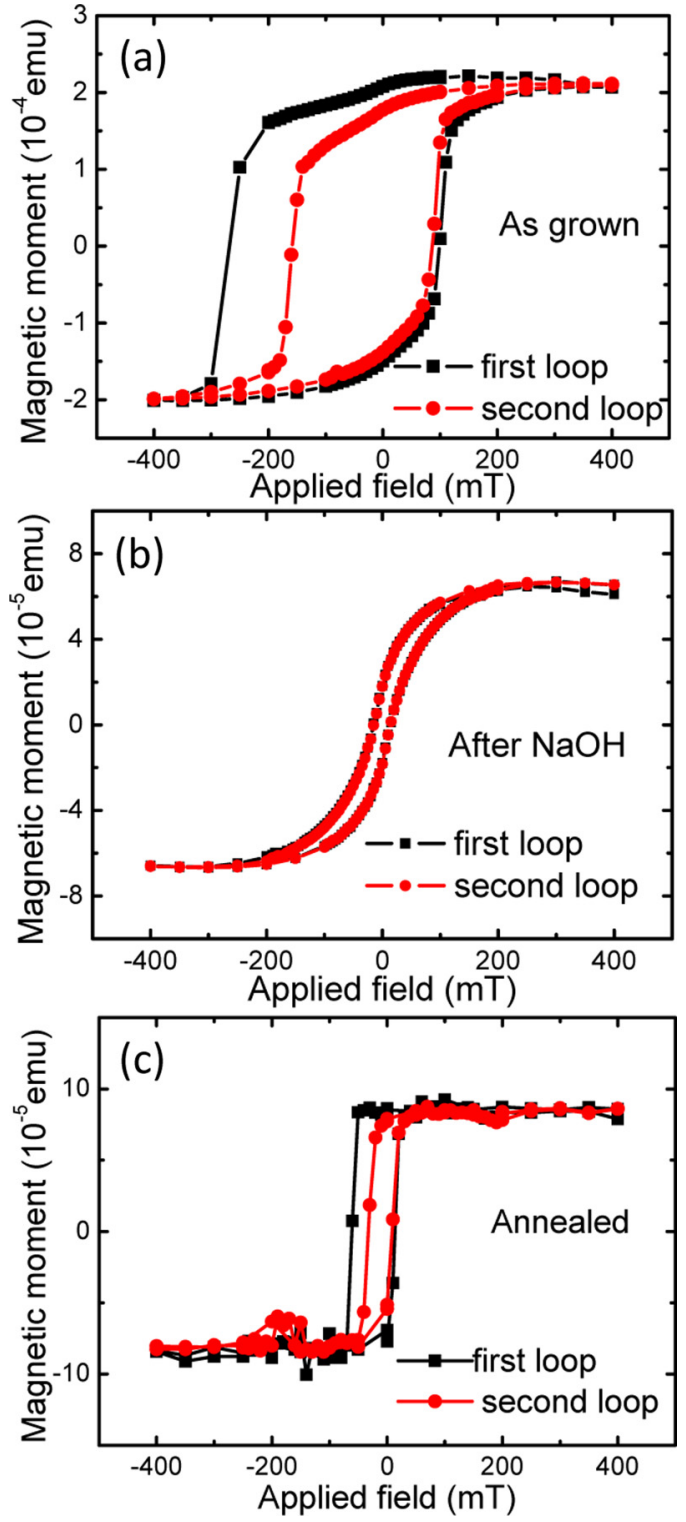

FIG. 4. (Color online) The first two hysteresis loops measured by SQUID magnetometry along the [100] direction of the $\mathrm{MgO}$ (100) substrate after field cooling for (a) the as-grown Co film, (b) this Co film after treatment with $\mathrm{NaOH}$ solution, and (c) this same Co film after annealing.

the UMA, which is superimposed on the fourfold symmetry in the as-grown magnetic thin films, is still under debate. In our case we can exclude anisotropic surface roughness as a possible origin [see the AFM topography image in Fig. 1(a)]. The UMA can be possibly attributed to Néel type interface symmetry breaking [4], which disappears in the treated Co films due to the newly created interface. It should be mentioned that there is no $\mathrm{Co}$ or $\mathrm{CoO}$ left after treatment in the bottom area between hexagonal nanodisks measured by scanning Auger microscopy (data not shown). The magnetization reversal in the treated Co film probably proceeds by magnetization rotation.

Figure 4(a) presents the result of the SQUID magnetization measurement of the $\mathrm{EB}$ effect along the $\mathrm{MgO}$ [100] direction

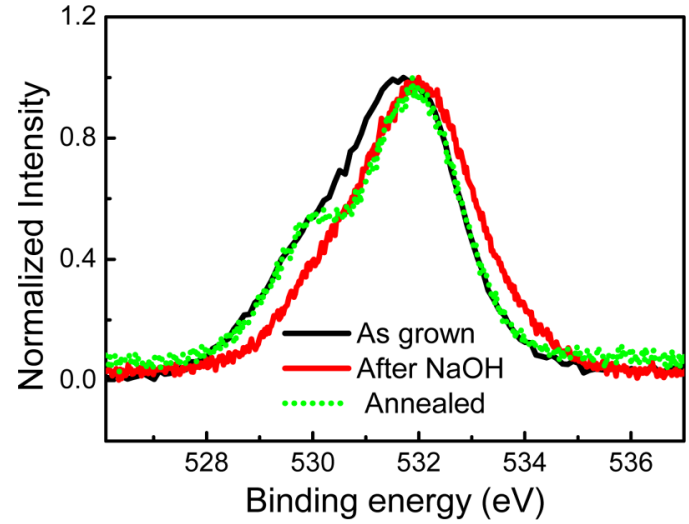

FIG. 5. (Color online) XPS high-resolution O $1 s$ spectrum for the as-grown Co film on $\mathrm{MgO}$ (100), for this Co film after treatment with $\mathrm{NaOH}$ solution, and for this same Co film after annealing.

in the as-grown Co film. After field cooling, the coercive field and the EB fields (shift of the center of the hysteresis loop) for the first and the second hysteresis loops are 175 and $119 \mathrm{mT}$ and 97 and $51 \mathrm{mT}$, respectively. The EB effect and the related training effect (a decrease of the coercive field and of the EB field when cycling through consecutive hysteresis loops) has already been extensively studied before [16]. The coercive fields inferred from the hysteresis loop are much larger than in polycrystalline $\mathrm{CoO} / \mathrm{Co}$ bilayers due to the influence of the magnetocrystalline anisotropy. After the treatment with the $\mathrm{NaOH}$ solution, the hysteresis loops reveal a disappearance of the EB effect as well as a drastic decrease of the coercive field to $15 \mathrm{mT}$ and of the total magnetic moment. The decrease of the coercive field and of the total magnetic moment can be related to the removal of the $\mathrm{Co}$ which is etched by the $\mathrm{NaOH}$ solution. The disappearance of the EB effect implies the removal of the $\mathrm{CoO}$ top layer accompanied by a chemical reaction between the $\mathrm{Co}$ and the $\mathrm{NaOH}$. In addition, the relatively small ratio $M_{r} / M_{S}$ between the remanent magnetization and the saturation magnetization is probably due to an extra contribution of $\mathrm{Co}(\mathrm{OH})_{2}$ [20]. The disappeared EB effect can be restored by the annealing process, as illustrated in Fig. 4(c). After the annealing the coercive fields and EB fields of the two hysteresis loops after field cooling are 37 and $22 \mathrm{mT}$ and 25 and $13 \mathrm{mT}$, respectively. This hints towards the formation of a different antiferromagnetic/ferromagnetic interface, which can be related to the transformation of $\mathrm{Co}(\mathrm{OH})_{2}$ into $\mathrm{Co}_{3} \mathrm{O}_{4}$ [14,22]. The antiferromagnetic Néel temperature $\left(T_{N}=40 \mathrm{~K}\right)$ is well above the measurement temperature. We note that the magnetization reversal in the descending branch of the first loop is much sharper than in Fig. 4(a), suggesting a negligible influence of magnetocrystalline anisotropy on the restored EB effect.

To further confirm the changing presence of the cobalt oxide, XPS measurements were performed in order to investigate the chemical composition of the cobalt oxide. In Fig. 5 we present the high-resolution $\mathrm{O} 1 s$ spectrum. After the $\mathrm{NaOH}$ treatment the main peak shifts slightly to the higher binding energy side when compared to the peak in the as-grown film. This is consistent with Ref. [22], where the $\mathrm{O} 1 \mathrm{~s}$ binding energy for oxide ions $\mathrm{O}^{2-}$ is smaller (around $529.4 \mathrm{eV}$ ) than 
that for $\mathrm{Co}(\mathrm{OH})_{2}$ and $\mathrm{CoOOH}$ (higher than $531 \mathrm{eV}$ ). After annealing, the main peak shifts back due to the transformation of the $\mathrm{Co}(\mathrm{OH})_{2}$ and $\mathrm{CoOOH}$. The additional peak corresponds to the characteristic peak of $\mathrm{Co}_{3} \mathrm{O}_{4}(530.0 \mathrm{eV})$ [22]. It can thus be concluded that the $\mathrm{NaOH}$ treated sample exhibits a higher contribution of hydroxylic functionalities at the surface than the as-grown sample, whereas after annealing the main contribution of the oxide species at the surface comes from the $\mathrm{O}^{2-}$ [26]. The qualitative analysis of the $\mathrm{O} 1 s$ spectrum is in accordance with the analysis of the XRD results, which confirms the different cobalt compounds that appear and disappear during the treatment with $\mathrm{NaOH}$ solution and the subsequent annealing process.

\section{CONCLUSIONS}

In conclusion, we investigated in detail the significant changes of the magnetic properties that occur in Co films grown on $\mathrm{MgO}(100)$ as a result of a treatment with $\mathrm{NaOH}$ solution. The continuous Co films evolve into isolated hexagonal nanodisks of $\mathrm{Co}(\mathrm{OH})_{2}$ by the treatment. This results in the disappearance of the uniaxial magnetic anisotropy and the two-jump domain-wall motion magnetization reversal mechanism, which are replaced by pure fourfold magnetocrystalline anisotropy and magnetization rotation, respectively. On the other hand, the removal of the $\mathrm{CoO}$ top layer by the $\mathrm{NaOH}$ solution eliminates the EB effect, which can be restored by an appropriate annealing process due to the formation of a new $\mathrm{Co}_{3} \mathrm{O}_{4} / \mathrm{Co}$ interface.

\section{ACKNOWLEDGMENT}

H.L.L. and T.H. contributed equally to this work. This work has been supported by the Research Foundation - Flanders (FWO, Belgium), by the Concerted Research Action program (BOF KU Leuven, GOA/14/007), and by the Flemish Hercules Foundation.
[1] C. Gould, K. Pappert, G. Schmidt, and L. W. Molenkamp, Adv. Mater. (Weinheim, Germany) 19, 323 (2007).

[2] J. Nogués, J. Sort, V. Langlais, V. Skumryev, S. Suriñach, J. Muñoz, and M. Baró, Phys. Rep. 422, 65 (2005).

[3] Y. Shim and J. G. Amar, Phys. Rev. Lett. 98, 046103 (2007).

[4] Y. Z. Wu, C. Won, and Z. Q. Qiu, Phys. Rev. B 65, 184419 (2002).

[5] R. Moroni, D. Sekiba, F. Buatier de Mongeot, G. Gonella, C. Boragno, L. Mattera, and U. Valbusa, Phys. Rev. Lett. 91, 167207 (2003).

[6] J. H. Gao, Y. Girard, V. Repain, A. Tejeda, R. Belkhou, N. Rougemaille, C. Chacon, G. Rodary, and S. Rousset, Phys. Rev. B 77, 134429 (2008).

[7] H. L. Liu, W. He, Q. Wu, H. T. Yang, X. Q. Zhang, and Z. H. Cheng, AIP Adv. 3, 062101 (2013).

[8] Q.-F.Zhan, S. Vanderzande, K. Temst, and C. Van Haesendonck, Phys. Rev. B 80, 094416 (2009).

[9] J. Nogués and I. Schuller, J. Magn. Magn. Mater. 192, 203 (1999).

[10] F. Radu, M. Etzkorn, R. Siebrecht, T. Schmitte, K. Westerholt, and H. Zabel, Phys. Rev. B 67, 134409 (2003).

[11] M. Gruyters and D. Riegel, Phys. Rev. B 63, 052401 (2000).

[12] H. Jung and A. Alfantazi, Corrosion 66, 035002 (2010).

[13] R. S. Jayashree and P. Vishnu Kamath, J. Mater. Chem. 9, 961 (1999).
[14] Z. P. Xu and H. C. Zeng, J. Mater. Chem. 8, 2499 (1998).

[15] Q. F. Zhan, S. Vandezande, C. Van Haesendonck, and K. Temst, Appl. Phys. Lett. 91, 122510 (2007).

[16] S. Brems, K. Temst, and C. Van Haesendonck, Phys. Rev. Lett. 99, 067201 (2007).

[17] X. G. Zhang and W. H. Butler, Phys. Rev. B 70, 172407 (2004).

[18] T. Gredig, I. Krivorotov, and E. Dahlberg, J. Appl. Phys. 91, 7760 (2002).

[19] O. Benamara, E. Snoeck, T. Blon, and M. Respaud, J. Cryst. Growth 312, 1636 (2010).

[20] P. Shamba, R. Zeng, J. Q. Wang, and S. X. Dou, J. Appl. Phys. 107, 09A919 (2010).

[21] Y. Nukaga, M. Ohtake, M. Futamoto, F. Kirino, N. Fujita, and N. Inaba, IEEE Trans. Magn. 45, 2519 (2009).

[22] J. Yang, H. Liu, W. N. Martens, and R. L. Frost, J. Phys. Chem. C 114, 111 (2010).

[23] Y. Zhu, H. Li, Y. Koltypin, and A. Gedanken, J. Mater. Chem. 12, 729 (2002).

[24] S. Yan, R. Schreiber, P. Grunberg, and R. Schafer, J. Magn. Magn. Mater. 210, 309 (2000).

[25] R. P. Cowburn, S. J. Gray, and J. A. C. Bland, Phys. Rev. Lett. 79, 4018 (1997).

[26] G. Carons, M. Nassir, and M. Langell, J. Vac. Sci. Technol. A 14, 1637 (1996). 